\title{
A Study on the Relationship Between Group Citizenship Behavior and Service Innovation Performance
}

\author{
Huang Ying ${ }^{1}$, Chen Chunni ${ }^{2}$, Wang Jing ${ }^{1}$ \\ ${ }^{1}$ School of Business Management, Guangxi University, Nanning, China \\ ${ }^{2}$ Guangxi Power Grid Co., Ltd, Liuzhou, China
}

Email address:

sunnygl@gxu.edu.cn (Huang Ying)

\section{To cite this article:}

Huang Ying, Chen Chunni, Wang Jing. A Study on the Relationship Between Group Citizenship Behavior and Service Innovation Performance. International Journal of Economic Behavior and Organization. Vol. 9, No. 3, 2021, pp. 94-102. doi: 10.11648/j.ijebo.20210903.17

Received: July 31, 2021; Accepted: August 24, 2021; Published: August 30, 2021

\begin{abstract}
Service innovation is a fundamental method for service enterprises to enhance dynamic capability in building core competitive advantage and achieving sustained growth as well. The improvement of service innovation performance requires enterprises to have more open cooperation vision, and flexible coordination mechanism as well. More focuses have steadily changed from the traditional machine-driven standardized process to flexible talent development of work teams, especially the improvement of their service innovation performance. It requires groups to show more team spirit, as well as mutual sharing behavior, which is beyond duty and not for the purpose of commendation. Based on the mediating effect of organizational commitment and knowledge sharing, this paper explored the effect of group citizenship behavior on service innovation performance in Chinese context. With a sample of 498 employees of enterprises in China, this paper explored the relationship between group citizenship behavior, service innovation performance, organizational commitment and knowledge sharing by constructing a structural equation model. The results showed that organizational commitment and knowledge sharing played a partial mediating role between group citizenship behavior and service innovation performance. It is recommended to establish the employee centered enterprise culture and regulations; foster an open atmosphere which is conducive to communication and innovation; establish a fair and effective incentive distribution system; establish a diversified knowledge sharing effective incentive system and give full play to the role of learning organization.
\end{abstract}

Keywords: Group Citizenship Behavior, Service Innovation Performance, Knowledge Sharing

\section{Introduction}

Global market is based on service competition rather than product competition gradually. With service innovation to improve service process and quality, service enterprises develop new products which make their core competitive advantage. It means that service innovation is a fundamental method for service enterprises to enhance their dynamic capabilities in building core competitive advantage and achieving sustained growth as well. The improvement of service innovation performance requires enterprises to have more open cooperation vision, and flexible coordination mechanism as well. Therefore, more focuses have steadily changed from the traditional machine-driven standardized process to flexible talent development of work teams, especially the improvement of their service innovation performance [1]. It requires groups to show more team spirit, as well as mutual sharing behavior, which is beyond duty and not for the purpose of commendation. It is defined as group citizenship behavior (GCB), which is described as a series of dedicated, spontaneous, mutual aid behaviors in a group level for the development of enterprise. Moreover, group citizenship behavior has been proved to contributes a lot to enhance organization performance [2-7]. Working in a complex mutual- aid environment with professional knowledge and skills, members of groups can consult and make use of colleagues' valuable ideas. In this way, organizational commitment and other incentives are strengthened, knowledge sharing and team communication increase faster which is conducive to innovative practices in service enterprises.

However, what is the essence of group citizenship behavior (GCB)? What is the impact of GCB on service innovation performance? How it exerts influence on service innovation 
performance? Furthermore, what kinds of measures should service enterprises take to develop GCB? These problems have not been well revealed in the existing studies. In order to establish a model of relationship between group citizenship behavior and service innovation performance, this paper selects the service enterprises in China to carry out the investigation and explores the effect. Thereafter, this paper makes suggestions for service enterprises. The research tries to expand the relevant research taking group citizenship behavior as antecedents and enrich the relevant research of promotion approach for service innovation performance as well.

\section{Theoretical Model Construction}

\subsection{Variable Definition}

\subsubsection{Group Citizenship Behavior}

Lv Zhengbao (2010) enriched the connotation of "group level organizational citizenship behavior" and named it "group citizenship behavior" [8]. This paper draw lessons from the definition which Lv Zhengbao put forward in 2010. It believes that group citizenship behavior regard the working groups in the organization as a whole that implement citizen behavior. It aims at achieving the overall goals of the organization, which is described as an extra role behavior. According to the existing research, this paper divides group citizenship behavior into three dimensions: organizational loyalty, team cooperation and helping behavior.

\subsubsection{Knowledge Sharing}

Researches before has not reached an agreement on the definition of knowledge sharing. This paper defines knowledge sharing as a process that the knowledge provider outputs, delivers, and spreads knowledge while the knowledge receiver inputs, absorbs, and internalizes knowledge. With this continuous process of interaction, knowledge sharing can maximize the utility of knowledge and improve innovation performance [9].

\subsubsection{Organizational Commitment}

Organizational commitment is known as the sense of belonging to the organization. Basis on three dimensional theory [10], this paper divides organizational commitment into two dimensions: affective commitment and continuance commitment. Affective commitment is the willingness to stay within the organization which is influenced by a number of factors, including the individual, job characteristics, experiences, and organizational structure as well. Continuing commitment is the perception of employees about losses if they leave the organization, including the benefits provided by the organization. In other words, when employees leave the organization, the potential resources and opportunities for careers might be reduced.

\subsubsection{Service Innovation Performance}

Moller, Rajala \& Westerlund (2008) found that service innovation can improve operating efficiency and create customer value. Brown (2013) insist a service innovation mode based on customer performance promotion. Therefore, innovation performance includes innovation enhance ability (Olimpia\&Racela, 2014), customer service satisfaction (Nachiappan Subramanian, 2014) and marketing performance (Chih-Wen Wu, 2014) as well [11-14]. According to Sun Ying (2009), this paper defines Service innovation performance as the index of enterprise's service innovation level and service innovation activity's completion effect. In order to measure the performance of service innovation completely, service innovation indicators should not only be judged by short-term objectives (e.g. financial performance), but also considered as the completion of the medium and long-term objectives with customers. Therefore, this study argues that service innovation performance should be divided into financial measurement indicators, customer metrics and internal measurement indicators.

\subsection{Theoretical Hypothesis}

\subsubsection{The Hypothesis of the Relationship Between GCB and Service Innovation Performance}

Qian Yuanyuan (2010) explored the impact of role behavior on organizational innovation performance at the individual level, and established relevant mechanisms. She found that different types of behavior may have direct or interactive effects on innovation performance. Cooperation and communication between groups will be smoother, When Staff and work groups in a higher sense of identity and loyalty which actively maintains company reputation and interests. Thereafter, service innovation performance may be improved. When employees and groups cooperate smoothly, the improvement of their own construction can encourage team morale, which has positive significance to service innovation performance. In the process of work, working groups encourage and care for each other, improve their feelings, and then enhance cohesion and improve efficiency of innovation activities. Based on the above analysis, this study considers that group citizenship behavior can positively contribute to service innovation performance, and makes the following hypothesis:

H1: There is a significant positive correlation between group citizenship behavior and service innovation performance;

H1-1: There is a significant positive correlation between the dimensions of group citizenship behavior and service innovation performance.

\subsubsection{The Mediating Effect of Knowledge Sharing and Organizational Commitment}

\section{(i) The Mediating Effect of Knowledge Sharing}

Knowledge sharing is a kind of interactive activity of information resources, which occurs in the context of social networks, thus the effectiveness of knowledge sharing is constrained by the social context. Hsu and Lin (2008) argue that organizational citizenship behavior helps people build strong relationships with each other, which encourages people 
to cooperate and help spontaneously. It can be concluded that employees with organizational citizenship behavior have more strong motivation and willingness to share knowledge, and more willing to participate in group activities. It helps to motivate staff to communicate, forms ideological collisions, and develop new ideas as well. Those group activities can be called a platform for sharing knowledge [12]. Davenport, T and Pnrsak, L points out that knowledge sharing helps work groups to quickly acquire knowledge about innovation, rapidly improves the creative ability of the working group, and improves organizational innovation performance [13]. Based on the previous theoretical foundations, the following hypotheses are proposed in this study:

$\mathrm{H} 2$ : Knowledge sharing is a mediator between group citizenship behavior and service innovation performance;

$\mathrm{H} 2-1$ : There is a significant positive correlation between the whole and the dimensions of group citizenship behavior and knowledge sharing;

H2-2: There is a significant positive correlation between the whole and the dimensions of knowledge sharing and service innovation performance;

H2-3: All dimensions of knowledge sharing play a mediating role between group citizenship behavior and service innovation performance.

\section{(ii) The Mediating Effect of Organizational Commitment}

In the empirical study, a large number of studies have proved that there is a significant positive correlation between organizational citizenship behavior and organizational commitment. At the same time, organizational commitment is an important factor affecting corporate performance. This paper believes that organizational commitment can be used to explain the perceived impact of group atmosphere and it is an internal process of generating innovative behavior and enhancing innovation performance. Therefore, this study proposes that organizational commitment is a mediating variable between group citizenship behavior and service innovation performance.

H3: organizational commitment is the mediator between group citizenship behavior and service innovation performance;

H3-1: the whole and dimensions of group citizenship behavior are positively related to organizational commitment;

H3-2: the whole and dimensions of organizational commitment are significantly positively related to service innovation performance;

H3-3: he whole and dimensions of organizational commitment play a mediating role between group citizenship behavior and service innovation performance.

\subsection{The Relationship Model Between Group Citizenship Behavior and Service Innovation Performance}

As an independent variable, GCB has a positive impact on service innovation performance, organizational commitment and knowledge sharing. Organizational commitment and knowledge sharing have positive effects on service innovation performance respectively. And then, we constructed a relational model, as shown in Figure 1. Through the model, we can find the relation between four variables: group citizenship behavior is the antecedents, service innovation performance is the result variable, and knowledge sharing and organizational commitment are mediating variables.

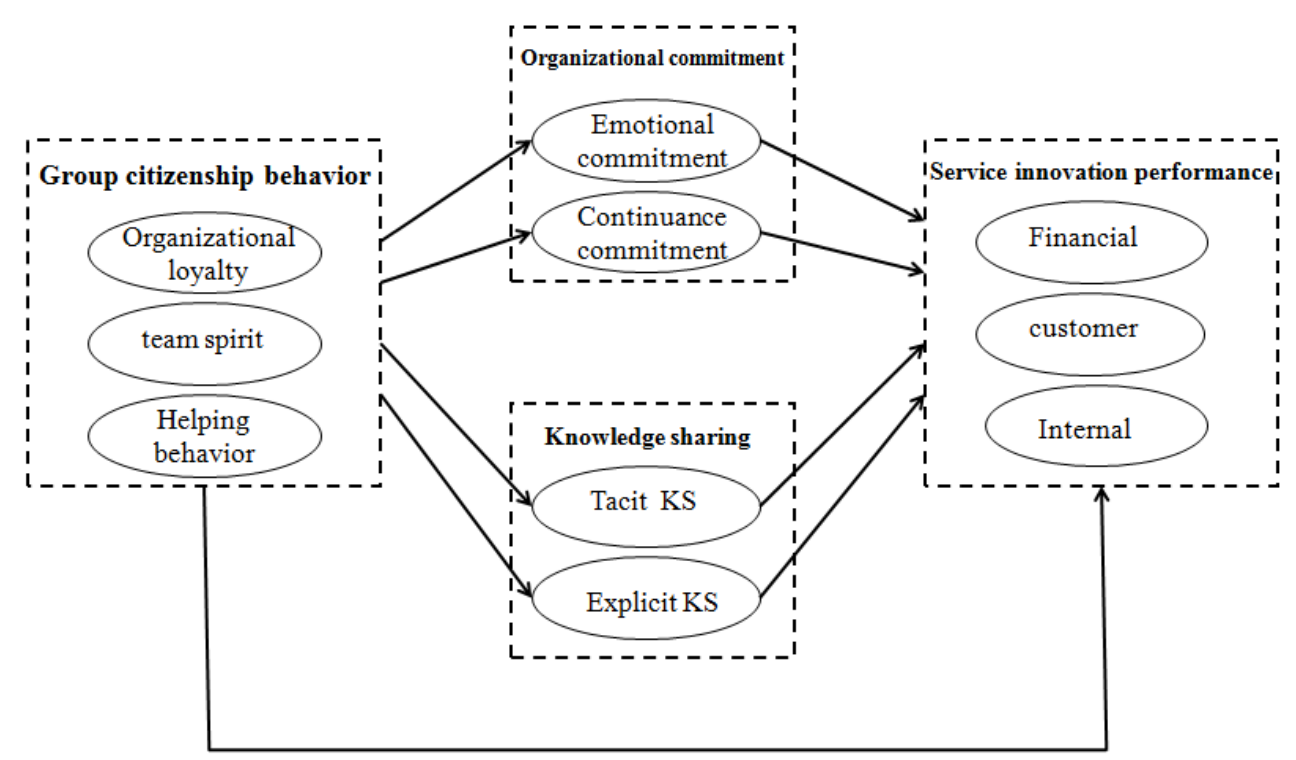

Figure 1. Model of relationship between group citizenship behavior, knowledge sharing, organizational commitment and service innovation performance.

\section{Empirical Analysis}

\subsection{Sample Selection and Data Collection}

Through the pre investigation, the questionnaire survey items were adjusted, and 600 questionnaires were issued at the formal investigation stage. The effective questionnaires were 498 , and the effective recovery rate was $83 \%$. In the survey, most of the respondents were young people, and the proportion of people under 35 years of age was as high as 
$65.3 \%$. About $1 / 3$ of them were under the age of 25 . The education level of the subjects was relatively high; most subjects are in the undergraduate and specialist level, and the proportion of subjects in these two education level were $76.9 \%$. Most of the surveyed departments or teams have more than 10 people, accounting for $47.79 \%$. The number of departments or teams in the investigation set up more than two years accounting for $65.46 \%$. In addition, most of the teams or departments involved in the investigation were homogeneous teams, accounting for $57.2 \%$. The most involved enterprises are state-owned or state-owned enterprises, accounting for nearly $1 / 3$, followed by private enterprises $(27.72 \%)$ and institutions $(23.69 \%)$.

\subsection{Concept Measurement}

We use object transfer consensus model to consider the design of questionnaire questions. All questions about the group citizenship behavior scale, the knowledge sharing scale and the organizational commitment scale were questioned by "my team". And then, calculate the average scores of individual members and aggregate them into group level for statistical analysis.

\subsubsection{Measurement of Group Citizenship Behavior}

The research carried out in this paper combines the arguments of many scholars, such as Lv Zhengbao (2010), Wu Xin, etc. (2005), Chen Xiaoping (2005) and Huang Ying [15], and mainly refer to Huang Ying's research, use of organizational loyalty, team spirit and helpful behavior in three different dimensions to explain group citizenship behavior. Therefore, the scale screened out 13 measuring items through the factor analysis and correlation analysis.

\subsubsection{Measurement of Knowledge Sharing and Organizational Commitment}

Bock, et, al. (2004) and Liu Jing (2008) have proposed a respectively mature and reliable method about the structure of knowledge sharing. The reliability and validity of this method has been tested and has a comprehensive summary. However, there are still some shortcomings in the scale. As the questions presented are too detailed and lengthy, it is easy to make the patient lose patience to fill out the questionnaire. Therefore, this study considers the opinions and suggestions which obtained from interviews, and optimizes the items.

At present, the most popular research model is a three-dimensional structure model which divided organizational commitment into affective commitment, continuous commitment and normative commitment. However, the existing models also have some problems that cannot be ignored, such as: emotional commitment and continuous commitment are differentiated well. There are also concepts and items overlap between continuous commitment and normative commitment. In addition, some studies have shown that normative commitment has little effect on innovation performance. Therefore, this study refers to the Organizational Commitment Questionnaire Revised by Luo Liang which has good reliability and validity. We screened and deleted the items, focusing on the two dimensions of affective commitment and continuance commitment of organizational commitment, excluding the items of normative commitment. After the test, 10 items are got.

\subsubsection{Measurement of Service Innovation Performance}

The service innovation performance measurement scale includes three aspects: financial indicators, customer indicators and internal indicators. It is developed by Hoegl\& Gemuenden (2001) and Huang, Chin \& Jiang (2008), choose the items from the prototypical scale, modify the items according to the object migration rules, and then form the service innovation performance scale. After analyzing the 8 items, we find that this scale has high reliability and validity.

\subsection{Research Method}

This study uses SPSS20.0 and AMOS23.0 to analyze and verify the relationship between group citizenship behavior and service innovation performance.

\subsubsection{Reliability and Validity of Variables}

With analyzing the reliability of scales of above variables, the results were shown in table 1. According to the results, we can find that the Cronbach a of all variables are greater than 0.8 , which shows that the reliability of all the scales are well.

Table 1. Reliability Group Citizenship Behavior, Knowledge Sharing, Organizational Commitment, Service Innovation Performance Measurement Scale.

\begin{tabular}{lll}
\hline Scale & Items & Cronbach a \\
\hline Group citizen behavior scale & 13 & 0.837 \\
Organizational commitment scale & 11 & 0.89 \\
Knowledge sharing scale & 10 & 0.89 \\
Service innovation performance scale & 8 & 0.837 \\
\hline
\end{tabular}

In this paper, we use AMOS23.0 to do confirmatory factor analysis and the three theoretical measurement model, and judge the rationality of the model by goodness-of-fit in table 2 .

Table 2. Model Suitability Test of Group Citizenship Behavior, Organizational Commitment and Knowledge Sharing.

\begin{tabular}{|c|c|c|c|c|c|c|c|c|}
\hline Model & $x^{2}$ & $d f$ & GFI & RMR & NFI & IFI & CFI & RMSEA \\
\hline Evaluation Criterion & - & - & $>0.9$ & $<0.05$ & $>0.9$ & $>0.9$ & $>0.9$ & $<0.08$ \\
\hline Group citizen behavior & 139.874 & 62 & 0.924 & 0.027 & 0.945 & 0.961 & 0.961 & 0.078 \\
\hline Organizational commitment & 153.841 & 34 & 0.926 & 0.042 & 0.941 & 0.953 & 0.953 & 0.075 \\
\hline Knowledge sharing & 136.254 & 43 & 0.897 & 0.047 & 0.908 & 0.923 & 0.923 & 0.087 \\
\hline
\end{tabular}

It can be seen that the fitness index of three-dimensional model of group citizenship behavior and the two-dimensional model of organizational commitment are up to standard. It shows that the model has a good fitting degree. In knowledge sharing model, the index GFI (0.897) and RMSEA (0.087) are not up to the standard, but quite close. So the two-dimensional 
model is ideal.

\subsubsection{Hypothesis Verification}

We used SPSS20.0 to analyze the relevance between variables. The correlation coefficients are shown in tables 3 and 4.
Table 3. Correlation of variables at the overall level.

\begin{tabular}{lllll}
\hline Variable & $\mathbf{1}$ & $\mathbf{2}$ & $\mathbf{3}$ & $\mathbf{4}$ \\
\hline 1. Group citizenship behavior & 1 & & & \\
2. Knowledge sharing & $0.486^{* *}$ & 1 & & \\
3. Organizational commitment & $0.496^{* *}$ & - & 1 & \\
4. Service innovation performance & $0.586^{* *}$ & $0.532^{* *}$ & $0.532^{* *}$ & 1 \\
\hline
\end{tabular}

Table 4. Correlation of variables at the dimension level.

\begin{tabular}{|c|c|c|c|c|c|c|c|c|}
\hline Variable & 1 & 2 & 3 & 4 & 5 & 6 & 7 & 8 \\
\hline 1. Helping behavior & 1 & & & & & & & \\
\hline 2. Organizational loyalty & $0.457^{* *}$ & 1 & & & & & & \\
\hline 3. Team spirit & $0.459^{* *}$ & $0.600^{* *}$ & 1 & & & & & \\
\hline 4. Explicit knowledge sharing & $0.275^{* *}$ & $0.389^{* *}$ & $0.437^{* *}$ & 1 & & & & \\
\hline 5. Tacit knowledge sharing & $0.366^{* *}$ & $0.359^{* *}$ & $0.378^{* *}$ & $0.688^{* *}$ & 1 & & & \\
\hline 6. Emotional commitment & $0.275^{* *}$ & $0.389^{* *}$ & $0.437^{* *}$ & - & - & 1 & & \\
\hline 7. Continuous commitment & $0.366^{* *}$ & $0.359 * *$ & $0.378^{* *}$ & - & - & $0.688^{* *}$ & 1 & \\
\hline
\end{tabular}

According to the data in the table, we can see that the correlation research results basically verify the theoretical hypothesis of this paper.

This study continues to construct the whole model through AMOS23.0 to verify the relationship between the four variables in the hypothetical model. The standardized results are shown below.

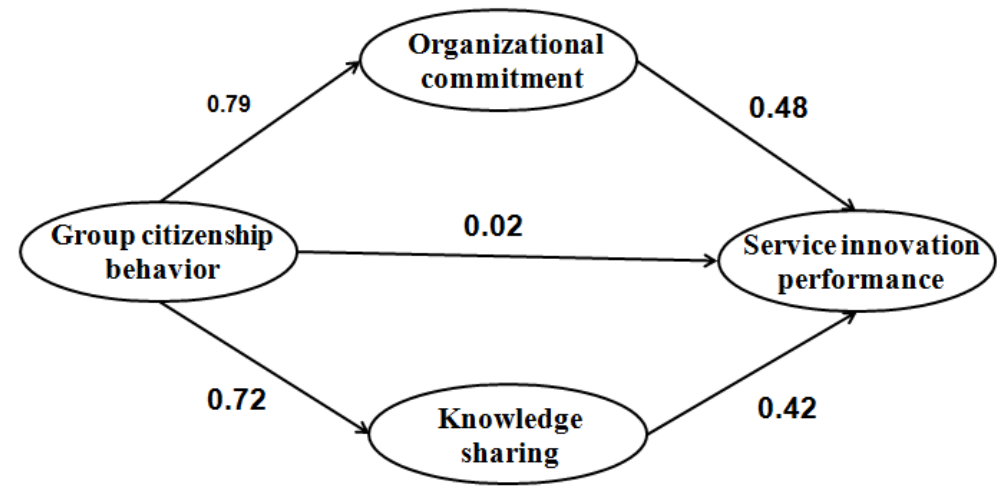

Figure 2. The relationship between group citizenship behavior, service innovation performance and their mediator variables at the whole level.

Normally, the fitting effect of the model can be judged by RMSEA, GFI, CFI and other indexes. RMSEA $<0.08$ (the smaller the better) while GFI, CFI $>0.9$ (the bigger the better). The fitting indexes of the model presented in this paper are shown in table 5. It can be seen that each fitting index of the model is appropriate. Therefore, the model fits well and can be used to verify the assumptions we have made, and the results are accurate.

Table 5. Test of Model Fitness.

\begin{tabular}{|c|c|c|c|c|c|c|c|c|c|}
\hline Model & $x^{2}$ & $d f$ & $x^{2} / d f$ & GFI & RMR & NFI & IFI & CFI & RMSEA \\
\hline Evaluation Criterion & - & - & $<3$ & $>0.9$ & $<0.05$ & $>0.9$ & $>0.9$ & $>0.9$ & $<0.08$ \\
\hline Test Model & 2082.935 & 807 & 2.581 & 0.889 & 0.044 & 0.937 & 0.923 & 0.923 & 0.064 \\
\hline
\end{tabular}

\section{Research Conclusions and Prospects}

\subsection{Conclusions and Discussions}

\subsubsection{Variable Structure of Group Citizenship Behavior, Service Innovation Performance, Organizational Commitment and Knowledge Sharing}

This paper divides GCB into three dimensions: organizational loyalty, team spirit and helping behavior according to the research by Huang Ying (2012). This division method is proved to be more reliable and practical than other methods in the research.

Meanwhile, the measurement of service innovation performance is mainly measured by three indicators: financial indicators, customer indicators and internal indicators. In the confirmatory analysis of the study, it was confirmed that the findings based on this model had high reliability and validity.

In the same way, the research have further filtered and optimized the design of knowledge sharing scale. It is determined that knowledge sharing variables are expressed in two dimensions, namely, explicit and implicit knowledge sharing, which is a comprehensive division method. Finally, organizational commitment scale focuses on two dimensions 
of organizational commitment: affective commitment and sustained commitment. The reliability and validity of the 10 questions are good.

\subsubsection{Positive Relationship Between GCB and Service Innovation Performance}

Through the analysis of the correlation between the two variables of group citizenship behavior and innovation performance, this paper find that their correlation coefficient is 0.586 , which shows that there is a positive correlation between them. At the same time, $p<0.01$ showed significant correlation between the two variables at the 0.01 level, which is proved as the same as the other scholars. Therefore, according to this result, the research prove that group citizenship behavior including organizational loyalty, team spirit and helping behavior as well, can inspire innovation performance in some way. With organizational loyalty, the positive relationship between groups will be more stable. Team spirit and helping behavior can stimulate knowledge sharing. More core information sharing will inspire more innovation performance. Therefore, service enterprises tend to strengthening collaboration between work groups and within work groups to encourage them sharing more core information and help each other positively, which can inspire new ideas (product) more efficiently. If work groups are given appropriate incentives, they might be more willing to take performance of mutual sharing and supporting, which is beyond duty and not for the purpose of commendation as well. With GCB shaping and corporate culture building inside, internal friction between work groups will be reduced sharply. In the meantime, work groups communicate and share their experiences or information more actively. Furthermore, the creativity ability of the whole enterprise will be improved. Finally, service innovation performance will be improved naturally.

\subsubsection{The Mediating Effect Between GCB and Service Innovation Performance}

In this paper, knowledge sharing is divided into two types: implicit and explicit, so it is necessary to make a more thorough discuss in the research, explore how each dimension works on innovation performance, and whether the effects are significant direct impacts. Through the investigation, we find that both implicit and explicit knowledge sharing can predict the service innovation performance in the process of acting as intermediary factor. Thus, in the management practice of service enterprises, we should not only cultivate group citizenship behavior, but also focus on the interaction between groups and members, and promote knowledge sharing, so as to effectively improve service innovation performance.

Research has confirmed that there is a significant correlation between organizational commitment and firm performance. At present, most scholars are exploring how organizational commitment affects the overall performance of the company, but there are few studies on how service innovation performance is affected by organizational commitment. Some scholars involved in their research: such as Han Yi (2011). In his research, he assumed that there is a correlation between organizational commitment and performance, and regarded innovation performance as part of job performance. This study sets organizational commitment as a mediating variable affecting group citizenship behavior and service innovation performance, explores the relationship between group citizenship behavior, organizational commitment and service innovation performance, and verifies that group citizenship behavior can effectively promote the improvement of enterprise service innovation performance through organizational commitment. In addition, Han Yi (2010) believes that the dimension of organizational commitment has a negative impact on performance, that is, the high sustainability of organizational commitment is not conducive to improving innovation performance. The results of this study show that sustained commitment has a weak positive effect on firm service innovation performance rather than a significant negative effect.

The reason why the results of this study are inconsistent with previous studies is as follows: first, usually when the researchers study on the relationship between organizational commitment and performance, they believe the innovation performance is including in job performance, they mix innovation performance and work performance together, and do not consider innovation performance as a dimension alone, so the assumptions are different, the measurement method and model are different, the final results also different. Second, the main focus of this study is on the Beibu Gulf service enterprises. Which's data are significantly different from those of the high-tech industry. Thus, empirical results will be different. Third, in our research, more than $60 \%$ of respondents of the survey were under 35 years of old, and $1 / 3$ of them were under 25 years of old. That is to say, most of the respondents were born after 1980, and quite a few were born after 1990. Their values and perceptions of employment differ from those of the previous ones.

\subsection{Management Inspiration}

\subsubsection{Design Cross-sectoral Key Performance Indicators to Encourage Group Citizenship Behavior}

Design cross-sectoral key performance indicators for performance incentives, encourage mutual assistance and cooperation among groups, to cultivate a fair competition atmosphere among groups while improving communication awareness and trust among groups as well. When designing performance evaluation indicators for cross-sectoral projects, the principle of "flexibility" should be followed. First, ensure the accuracy and rationality of the assessment indicators, and pay attention to the coexistence of efficiency and effectiveness. When design evaluation indicators for performance of cross departmental projects, enterprises should set up "cross department project efficiency", "cross department project quality" and other related indicators for the relevant departments of the project, and link them with individual's evaluation and reward. Second, the assessment is comprehensive, not only to assess the results of the task, but also to pay attention to the process of assessment. In this process, project monitoring commissioners are appointed to 
follow up and communicate with each other on the progress of cross-departmental projects, and bring the project follow-up content and communication effect into the performance evaluation index.

\subsubsection{Establish Employee Centered Culture and Rules to Enhance Employee's Sense of Ownership}

Enterprise can create a cultural atmosphere with humanistic care, and encourage employees to participate in enterprise management. Comparatively speaking, the power distance of Chinese enterprises is relatively large, and it is easy for the superiors to ignore the needs of the subordinates to form a paternalistic leadership style, resulting in low loyalty of employees to the enterprise and low sense of organizational commitment. Therefore, in order to shape and cultivate group citizenship behavior, we should establish a staff-oriented cultural system, pay attention to the needs of employees, and create a humanized corporate culture atmosphere. In order to enhance the positive psychological perception of employees, so that employees feel the sense of concern, fairness and support of the enterprise, and stimulate Team Citizenship behavior. Firstly, rewards such as "Excellent Suggestion Award" should be offered to employees who put forward constructive suggestions. Employees are encouraged to make bold suggestions and suggestions, and actively participate in the construction and management of enterprises. Secondly, we should authorize employees appropriately, give them a certain scope of rights, and have the right to make appropriate decisions independently in their work. In the field which employees pay attention to and which is closely related to employees' interests, such as performance appraisal, reward policy, salary promotion, empower employees to negotiate with managers, and give full consideration to employee's opinions when making enterprise goals. In terms of departmental regulations, fully considering and listening to employees' needs and empowering employees to participate in the formulation of regulations can not only ensure employees' understanding and support of policies, but also reflect the concern and support of organizations for employees. Third, when authorized to employees, enterprises should pay attention to the gradual implementation of phased goals, to give employees continuous performance communication and guidance.

\subsubsection{Pay Attention to the Key Role of Managers in the Cultivation of Group Citizenship Behavior}

As the key to cultivating group citizen behavior, managers must demonstrate a high sense of responsibility and team spirit in their work to drive and guide group citizenship behavior. First, when selecting and configuring managers, pay attention to emotional intelligence and moral of the candidates, and choose those who is highly responsible, team oriented, passionate, self controlling and good at delivering positive energy as a manager. Second, conduct regular training for management and set up performance evaluation indicators for senior management, such as "supporting group work actively", "paying attention to interaction and communication between groups", and "positive evaluation by subordinates". Third, when managers are tolerant of employees' occasional mistakes, it might be beneficial for enterprises to cultivate a relatively relaxed, open and innovative atmosphere.

\subsubsection{The Principle of "Flexibility" Should Be Followed in Recruitment and Training}

Firstly, in the process of employee recruitment, the organization should take into account the characteristics and needs of employees and adopt flexible matching measures to stimulate employees' organizational trust and identity. Secondly, the training process of enterprises should also be managed flexibly: Attaching importance to the importance of training, changing traditional inculcating training methods to make training functions flexible; formulating rigorous training needs analysis. The training content not only involves the improvement of knowledge and skills, but also pays more attention to the cultivation of employees' values and attitudes.

\subsubsection{Give Full Play to the Positive Role of Knowledge Sharing}

It is an important prerequisite for knowledge sharing that each knowledge owner has the willingness to share knowledge. From the perspective of knowledge sharing, the knowledge and skills that employees master as their "private property" are essentially a complex game process in the process of sharing within the organization, and will not naturally occur. Only by taking appropriate incentives and incentives for knowledge owners, can knowledge sharing be promoted. If employees worry about losing their competitive advantage because of knowledge sharing, they will lack the enthusiasm of knowledge sharing. When employees think that knowledge sharing can match their efforts with their gains and even more, they would be willing to share their knowledge with others. Therefore, enterprises should establish a sound and mutually beneficial incentive mechanism, cultivate the habit of sharing knowledge, and realize the promotion of innovation performance.

Design cross departmental resource transfer mechanisms. Provide employees with resources and environments that are not available independently, prompting voluntary interaction and creative communication among departments. It is suggested that conditional enterprises or units develop information sharing and mutual assistance platform, integrate information and resources that can be disclosed by various departments, and share information and resources on this platform for downloading, learning and discussion by employees of various departments. For employees and departments who actively link knowledge, certain material and spiritual incentives are given.

At the same time, a mutually beneficial knowledge sharing mechanism should be established. Specific measures are as follows. First, implementing tutorial system which means that equipping senior staff as mentors for new staff when they are hired. The mentors are responsible for leading new employees to adapt to the organization as soon as possible and guiding them to complete their daily work. Second, listing the guidance achievement of senior staff as an indicator of performance appraisal, and senior staff may therefore be rewarded or promoted. These not only are positive for new 
employees, but also enhance the old staff's sense of self accomplishment and organizational identity, and stimulate their motivation of sharing of work experience.

Develop learning enterprise. When a group or organization is established solely to accomplish a task, employees may not continue to share knowledge and cannot maintain the habit of sharing knowledge due to the influence of interests after the task is completed. Therefore, in order to form a good knowledge sharing atmosphere within the enterprise, the following three measures can be taken. First, enterprises should build learning organizations and promote communication among employees and working groups. Second, enterprises can establish employee databases, understand employees' interests, expertise and development needs, set up associations and organizational activities to encourage cross department communication, and create a good platform for employees. Third, enterprises should set learning goals and evaluate the various departments of the exchange of learning achievement regularly. Enterprises could set up effective time management and organization management mechanism, divide the responsibilities clearly, and ensure the sustainable development of knowledge sharing.

\subsubsection{Give Full Play to the Positive Role of Organizational Commitment}

First, enterprises should link employees' rewards and punishments with the overall performance of their groups or projects as well, while promoting employees to act in favor of the team or organization. And the results of performance appraisal should be directly related to the salary and promotion of employees. The salary distribution system should be based on contribution to ensure relative fairness. Second, the performance appraisal and performance reward should be fair and impartial to employees and groups. The fair and just perception of performance appraisal and performance reward can influence the attitude and confidence of employees. Therefore, enterprises should carry out a scientific, transparent, fair and open assessment system, make employees recognize the enterprise system and resonate with the enterprise. Third, managers should carry out follow-up communication on performance appraisal, to help employees make improvement according to the evaluation results, and to avoid the "Stuffy bag" problem that performance evaluation results are not understood and recognized by employees, and failure to guide and optimize practical work.

Adopt flexible incentive mode, and foster trust and sense of identity of the employees, which enables employees to feel strong support and happiness in the working groups. In addition, employees will have confidence in their future career development and hope to stay in the organization for a long time. This sense of belonging will make employees show a stronger sense of responsibility. First, enterprises can provide employees with a variety of "personalized welfare package" for employees to choose which reflecting the humane care of enterprises. Second, enterprises can create a comfortable and safe working environment and relieve employees' working pressure. Third, enterprises should be targeted to develop training programs for employees' occupation career to cultivate long-term psychological contract between employees and enterprises. Making employees achieve personal development in the enterprise securely, and willing to contribute to enterprise development.

Enterprises should pay attention to the cultivation of collectivism values and the correct concept of fairness. When selecting employees, enterprises should pay attention to their ability of cooperation and communication, and choose those who are highly collective. At the same time, in the aspect of employee's ability improvement and training, enterprises should help employees to set up the concept of "equal opportunity" rather than "equal results", promote healthy competition among employees, and give full play to the incentive function of the salary system.

\section{References}

[1] Racela: Customer Orientation, Innovation Competencies, and Firm Performance: A Proposed Conceptual Model [J]. Social and Behavioral Sciences, (148): 16-23 (2014).

[2] Chen X. P, Lam S, Naumann S, et al: Group Citizenship Behaviour: Conceptualization and Preliminary Tests of its Antecedents and Consequences [J]. Management and Organization Review, 1 (2), 273-300 (2005).

[3] Schnake M, Dumler M: Level of Measurement and Analysis Issues in Organizational Citizenship Behavior Research [J]. Journal of Occupational and Organizational Psychology, (76), 283-301 (2003).

[4] Ehrhart M, Naumann S: Organizational citizenship behavior in work groups: a group norms approach [J]. Journal of Applied Psychology, 89 (6): 960 (2004).

[5] Euwema M C, Wendt H, Emmerik H V: Leadership styles and group organizational citizenship behavior across cultures $[\mathrm{J}]$. Journal of Organizational Behavior, 28 (8), 1035-1057 (2007).

[6] Shin Y, Jin N C: What makes a group of good citizens? The role of perceived group-level fit and critical psychological states in organizational teams $[\mathrm{J}]$. Journal of Occupational and Organizational Psychology, 83 (2), 531-552 (2010).

[7] Chou S Y, Garcia D C: Group Organizational Citizenship Behavior in the Stages of Group Development [J]. International Journal of Business \& Management, 6 (10), (2011).

[8] Lv Zhenbao, Lin Wenquan, Ma Chao: Review of Group Citizenship Behavior [J]. Economic Management of East China, 2, (2010).

[9] Wu Xiaobo, Gao Zhongshi, Wei Shijie. Empirical Study on Tacit Knowledge Externalization and technological innovation performance [J]. Scientific Research, 2007, 25 (6): 1233-1237.

[10] Meyer J. P., Allen N. J. Commitment to Organizations and Occupations: Extension and Test of a Three Component Conceptualization [J]? Journal of Applied Psychology, 78, 538-551 (1993).

[11] Cohen S G, Bailey D E: What makes teams work: Group effectiveness research from the shop floor to the executive suite [J]. Journal of Management: Official Journal of the Southern Management Association, 23 (3), 239-290 (1997). 
[12] Chih-Wen Wu: The study of service innovation for digiservice on loyalty [J]. Journal of Business. 67, 819-824 (2014).

[13] Nader Nada, Zulfiqar Ali. Service Value Creation Capability Model to Assess the Service Innovation Capability in SMEs [J]. Procedia CIRP 30, 390-392 (2015).

[14] Hooff, B. V \& Weenen, F. S: Committed to share: commitment and CMC use as antecedents of knowledge sharing [J]. Knowledge and Process Management, Jan-Mar, 11 (1), 13 (2004).

[15] Huang Ying: Cultivation of Group Citizenship Behavior: The Coupling Model of Strategic Human Resource Practice Based on Flexible Stimulation [J]. China Industrial Economics, 10, 122-133 (2012). 